\title{
La reconnaissance des acquis expérientiels: étude de cas de la politique d'admission des adultes à l'Université Laval
}

\author{
LUCIE HÉON*, RENÉE CLOUTIER et PIERRE W. BÉLANGER
}

\section{RÉSUMÉ}

Les auteurs évaluent formellement la politique et le processus d'évaluation de la clientèle adulte qui ne rencontre pas les critères "normaux" d'admission aux programmes de premier cycle à l'Université Laval en 1980.

L'étude de l'application de cette politique, tenant compte de l'expérience de vie ou des acquis expérientiels des candidats adultes en plus de leurs acquis scolaires antérieurs et l'analyse d' un échantillon de 400 dossiers de candidats adultes nous révèlent que l'âge et la durée de l'expérience constituent des facteurs favorisant l'admissibilité des candidats adultes. Elles démontrent aussi clairement que le profil de référence, lors de ce processus d'évaluation, est principalement la formation scolaire et sa dimension cognitive. En effet, le fait de posséder des expériences liées à l'apprentissage, au moins un diplôme de niveau collégial et des résultats scolaires supérieurs à $65 \%$, augmente les chances d'un candidat d'être reconnu admissible.

Le concept d'expérience de vie semble donc être utilisé de façon secondaire, comme confirmation au renforcement des succès scolaires antérieurs, plutôt que comme critère autonome.

\section{ABSTRACT}

Has the inclusion of experience as a criterion of admission for adult students raised significantly the chances of these students of being admitted?

The authors analyse the new admission policy at Laval University (1980) and, from a sample of 400 candidates' dossiers, its practice age and a fair amount of experience appear to be important factors opening the door of university. However, the effects of these factors are particularly enhanced if experience is close to academic or cognitive learning or combined with a CEGEP diploma and a grade higher than $65 \%$.

Thus, experience does not seem to be used as a basis of admission of its own, but mainly to corroborate academic ability already expressed by good grades.

*Lucie HÉON, étudiante au doctorat en administration et politique scolaires. Renée CLOUTIER et Pierre W. BÉLANGER sont sociologues et professeur-e-s titulaires en administration et politique scolaires.

Note: Dans la présente recherche, le générique masculin est utilisé sans aucune discrimination et uniquement dans le but d'alléger le texte. 


\section{INTRODUCTION}

Depuis le début des années 1960, les universités canadiennes ont élargi leurs critères d'admission pour faire place à une clientèle d'adultes qui ne rencontrait pas les critères dits "normaux" d'admission. La notion d'expérience de vie, d'acquis expérientiels est alors apparue dans les conditions d'admission des adultes. Mais comment cette nouvelle politique d'admission a-t-elle été appliquée? L'introduction de ces nouveaux critères a-t-elle ouvert la porte à un nombre important d'adultes? Jusqu'à date, ces questions sont demeurées sans réponse comme le soulignait récemment Darling (1985: 96). Notre recherche s'inscrit dans une tentative d'évaluation formelle de la politique et du processus d'admission de la "deuxième chance" dans une université québécoise francophone, Laval.

\section{Politique d'admission des adultes à l'Université Laval}

Au Québec, la Commission Parent avait attaché une grande importance dans son rapport (1963) à la notion d'éducation permanente et préconisé le développement des services d'éducation pour les adultes. A sa suite, le ministère de l'Education du Québec avait précisé les champs de responsabilité de l'éducation permanente aux niveaux d'enseignement secondaire et collégial mais avait laissé aux universités elles-mêmes la tâche de développer l'éducation des adultes au niveau universitaire.

En 1973, l'Université Laval accepte le principe de l'éducation permanente. Selon le directeur du Service d'accueil de l'époque, le choix fait par l'Université Laval pour institutionnaliser 1'éducation des adultes se résume ainsi:

- on ne veut pas de structure spéciale pour l'éducation des adultes, telle une faculté d'éducation permanente;

- on veut permettre à tous les adultes d'avoir accès à toutes les activités normales et régulières;

- on veut développer au besoin de nouvelles fonctions de services à la collectivité tels la création du Service d'accueil de l'adulte, le Service de l'éducation permanente et le Service de Counselling (Pelletier 1979:2).

Il faut préciser qu'avant l'adoption de ces principes en 1973, aucune politique spécifique n'existait à l'Université Laval dont l'objectif était l'organisation systématique de l'éducation des adultes. Il y avait bien eu, en 1938, une première session de cours d'été offerte à l'Université Laval, mais cette activité fut gérée jusqu'en 1972 par une entité administrative autonome s'autofinançant en bonne partie. Chacune des facultés et des écoles jouissait d'une liberté totale concernant l'éducation à offrir à la clientèle adulte et à la clientèle des jeunes.

\section{Service d'accueil des adultes}

Un des éléments de la stratégie d'implantation de l'éducation permanente fut la mise sur pied en 1973 du Service d'accueil des adultes. Ce Service est alors rattaché au vice-recteur à l'enseignement et à la recherche et il a pour objectif d'aider: 
- les adultes dans leurs démarches préalables à leur admission dans un programme de cours régulier à l'université;

- les étudiants (adultes et jeunes) qui, rencontrant des difficultés dans le cours de leurs études, ont besoin d'être orientés et, le cas échéant, d'être dirigés vers d'autres services dé l'Université (CUL,1973).

Aux fins de l'accès à ce Service, l'Université donne une définition opérationnelle de l'adulte. Est considéré comme adulte:

"la personne âgée de 21 ans qui a mis un terme à la fréquentation du système scolaire depuis au moins 24 mois au moment où elle soumet la demande d'admission et qui témoigne d'une expérience pertinente aux études qu'elle désire entreprendre" (CUL, 1974)

Pour les candidats qui ont connu des interruptions plus ou moins longues dans leurs cheminements scolaires, l'Université Laval a développé des mécanismes pour mesurer leur capacité de réussite aux études universitaires qu'ils désirent entreprendre. Entre autres, les responsables du Service d'accueil des adultes évaluent les dossiers du candidat adulte avant même que ce candidat puisse postuler une demande officielle d'admission dans un programme régulier.

Avant 1973, l'admission et l'évaluation du dossier de ces candidats pour les fins de l'admission au premier cycle relevaient des facultés. Ainsi, les facultés détenaient le pouvoir presque exclusif d'établir les critères d'admission et de décider du profil des individus qui pouvaient être admis à suivre leur enseignement.

Donc, bien plus qu'une simple redéfinition du statut de l'adulte, nous pouvons observer une volonté de la part de l'Université Laval, en 1973, de centraliser et d'uniformiser l'évaluation des dossiers des candidats adultes à un même service.

\section{Processus d'évaluation des dossiers des candidats adultes par le Service d'accueil}

Le processus d'évaluation des dossiers des candidats adultes par le Service d'accueil en 1980 se divise en trois grandes étapes:

1. Lorsqu'un adulte répond à la définition déjà donnée de l'adulte, il se présente au Service d'accueil pour y formuler son projet d'étude. Lors de cette rencontre, les conseillers déterminent la nature du projet, soit qu'il est d'ordre culturel ou de perfectionnement. Ils procèdent ensuite à l'évaluation des acquis de l'adulte à l'aide de la grille d'évaluation S.E.M. (Scolarité, Expérience, Motivation).

Cette grille d'évaluation pondère la scolarité $(S)$, l'expérience $(E)$ et la motivation $(\mathbf{M})$.

Les résultats de cette évaluation ne sont pas communiqués à l'adulte, ils demeurent cependant dans le dossier du candidat. $A$ la lumière des résultats obtenus, les conseillers établissent avec le candidat la liste des prérequis à remplir en fonction de son choix disciplinaire. Le cas échéant, le conseiller détermine les cours d'appoint de niveau collégial et les cours compensateurs - dispensés à l'Université - que le candidat doit suivre pour rencontrer les exigences d'admission au programme désiré.

2. Une fois les conditions d'admission remplies par le candidat, le Service 
d'accueil transmet au comité d'admission du programme choisi un avis sur l'admissibilité de cet adulte.

3. La dernière étape de ce processus d'admission se termine par l'offre de l'Université, c'est-à-dire par la réponse d'admission du comité d'admission en question. (Héon, 1984)

\section{Outil d'évaluation: la grille S.E.M.}

L'utilisation de la grille d'évaluation S.E.M. par le conseiller constitue l'étape principale du processus d'évaluation des chances de réussite aux études universitaires du candidat adulte. On y évalue quantitativement les acquis du candidat.

L'objectif principal de cette grille est, selon le Service d'accueil (1979),

"d'établir les chances de succès raisonnables d'un candidat. Ces objectifs sont:

- d'aider les candidats adultes à s'auto-évaluer selon les critères communs et, s'il y a lieu, à réviser leur projet d'études;

- d'arriver à mieux choisir le nombre et la nature des cours d'appoint qui conviennent à chaque candidat,

- d'aider les conseillers dans les démarches de l'entrevue;

- d'assurer l'homogénéité de langage des conseillers;

- de garantir un traitement comparable à tous les candidats".

Cette grille permet de classer provisoirement les candidats à des fins d'analyse de la clientèle et de formuler des opinions d'admissibilité (Service d'accueil, 1979: 1). Elle sert aussi à évaluer le candidat entre la première entrevue et la demande d'admission. Les conseillers peuvent en outre s'inspirer des résultats de cette évaluation pour justifier l'à-propos des cours d'appoint suggérés au candidat.

La formulation des opinions d'admissibilité par les conseillers se base entre autres sur le total des points obtenus lors de l'évaluation des acquis de l'adulte à l'aide de la grille d'évaluation S.E.M. (voir figure 1).

Un score inférieur à treize points attribué à un dossier est jugé dans l'ensemble faible. L'opinion d'admissibilité, émise dans ce cas, est associée à une candidature non admissible aux études universitaires. Dans la plupart des cas, le Service d'accueil des adultes peut conseiller aux candidats de suivre un certain nombre de cours d'appoint au Cégep. Le dossier du candidat sera réévalué lorsqu'il aura complété ces pré-requis.

Un score supérieur à treize points attribué à un dossier est jugé dans l'ensemble bon. L'opinion d'admissibilité, émise dans ce cas, est associée à une candidature admissible aux études universitaires. Dans certains cas, le Service d'accueil des adultes peut conseiller aux candidats de suivre quelques cours compensateurs à l'Université.

\section{Évaluation du processus d'admission}

Pour les fins de notre travail, nous avons choisi quatre cents (400) dossiers au hasard parmi l'ensemble de la population ayant fait appel au Service d'accueil en 1980. De ceux-ci, 229 avaient fait l'objet d'une notation selon la grille d'évalua- 


\section{DOULLE DPEVALUATIOM DEM}

\begin{tabular}{|c|c|c|}
\hline \multicolumn{3}{|c|}{ SCOLARITE } \\
\hline VECU & niveau de scolarité atteint & $(1-4)$ \\
\hline ACQUIS & moyenne obtenue & $(0-4$ \\
\hline
\end{tabular}

\section{EXPERIENCE}

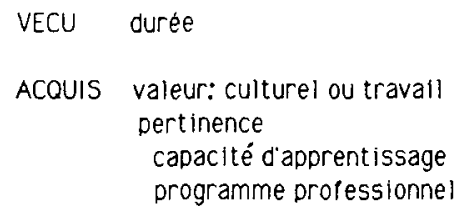

\section{MOTIVATION}

intensité

motifs invoqués
$(0-2)$

SOURCE : Service d'Accueil, Qrille d'éyaluation SEM nour l'éyaluation des ocouis, fevr ier 1979

tion S.E.M., notre variable dépendante, et ont été retenus pour l'analyse statistique.

La nature même des éléments sur lesquels repose l'articulation de la définition du candidat adulte met bien en évidence l'importance de l'âge d'un candidat pour que celui-ci soit considéré comme adulte et ainsi avoir accès au Service d'accueil. Le choix de la variable âge comme variable indépendante principale nous est apparu pertinent car le degré d'admissibilité semble être beaucoup plus influencé par l'âge que par le sexe (Héon, 1984: 89-94). Les variables contrôles sont, pour les acquis scolaires: le niveau scolaire et les résultats scolaires; pour les acquis expérientiels: la durée de l'expérience et la pertinence de l'expérience.

Les tests utilisés sont celui du Chi-carré $\left(\chi^{2}\right)$ et le coefficient de Pearson $(\mathrm{R})$. Le seuil de signification statistique retenu est plus petit ou égal à 0.05 ; l'intensité de l'association significative est égale ou supérieure à 0.25 . 
Tableau: I DISTRIBUTION DES CANDIDATS ADULTES SELON

IE DEGRE DAAMISSIBILITE ET L AGE

\begin{tabular}{|c|c|c|c|}
\hline AGE & \multicolumn{3}{|c|}{ DEGRE DADMISSIBILITE } \\
\hline 30 anst & $\begin{array}{c}\text { ADMISSIBLE ( }(8) \\
46,2\end{array}$ & $\begin{array}{c}\text { NON ADMISS IBLE (\$8) } \\
53,8\end{array}$ & $\begin{array}{c}n \\
130\end{array}$ \\
\hline 29 ans- & 12,9 & 87,1 & 155 \\
\hline \multirow[t]{2}{*}{ Total $\$$} & 28,1 & 71,9 & 285 \\
\hline & $x^{2}=37.09$ & $R=0,37$ & \\
\hline
\end{tabular}

Compte tenu de la définition du statut d'adulte à l'intérieur de la politique d'admission, l'âge semble être un facteur déterminant de l'admissibilité du candidat lors de l'évaluation de son dossier. Il y a tout lieu de croire que les individus les plus âgés ( 30 ans et plus) seront reconnus en plus grand nombre admissibles aux études universitaires.

L'age n'est toutefois pas la seule condition à remplir pour pouvoir être reconnu admissible. En effet, le candidat doit avoir interrompu ses études depuis un certain temps et posséder une expérience pertinente.

Parmi tous les candidats s'adressant au Service d'accueil, nous supposons que les candidats les plus âgés ( 30 ans et plus) auront une durée d'expérience plus longue que ceux de 29 ans et moins. Cependant, cette expérience ne sera pas forcément plus pertinente que celle des candidats jeunes ( 29 ans et moins). Selon ces hypothèses, la relation entre l'âge et le degré d'admissibilité devrait se maintenir, lorsque nous contrôlons par la durée de l'expérience, mais cette relation pourrait être modifiée en contrôlant par la pertinence de l'expérience.

A l'intérieur de la politique d'admission des adultes, il n'est en aucun cas précisé que le candidat doit posséder un niveau scolaire précis (ex. D.E.C. ou D.E.S.) ni de résultats scolaires particuliers. Dans ce cas, la relation entre l'âge et le degré d'admissibilité devrait se maintenir lorsque nous la contrôlons par les acquis scolaires des candidats. Ainsi, l'âge constituerait le facteur principal de l'admissibilité. Qu'en est-il dans les faits?

\section{Résultats}

L'âge semble être un facteur déterminant du degré d'admissibilité des candidats. adultes. En effet, bien que la majorité des candidats soit jugée non-admissible (71.9\%), les candidats reconnus admissibles se retrouvent en plus grand nombre lorsqu'ils ont 30 ans et plus (tableau 1).

Cependant, l'âge n'est plus un facteur déterminant du degré d'admissibilité des candidats adultes lorsque nous contrôlons par la variable durée de l'expérience (tableau 2). 


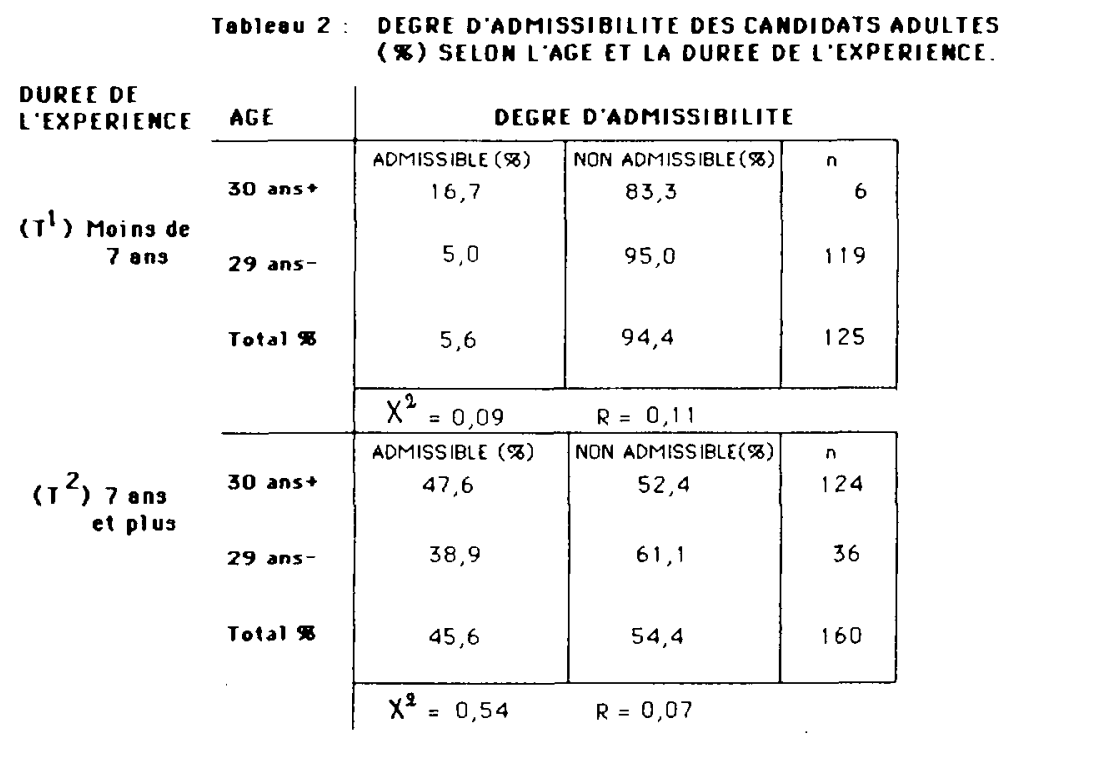

Tableau 3: DEGRE DADMISSIBILITE DES CAMDIDATS ADULTES (\$) SELON L'AGE ET LA PERTINENCE DE L'EXPERIENCE

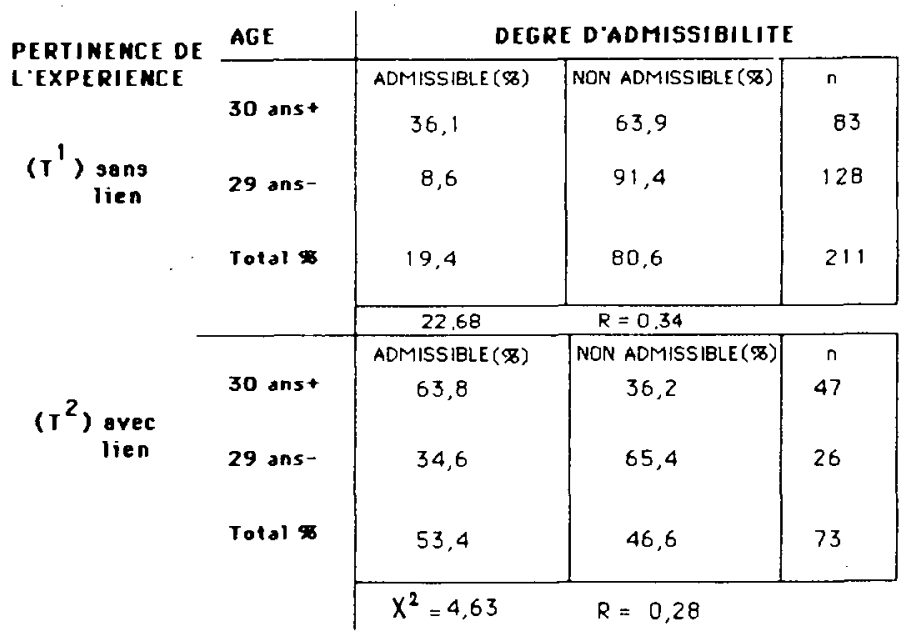

En contrôlant cette fois la relation entre l'âge et le degré d'admissibilité par la pertinence de l'expérience, nous observons d'une part que, pour les candidats dont l'expérience est évaluée sans lien avec l'apprentissage, l'âge demeure un facteur favorisant l'admissibilité; d'autre part, lorsque l'expérience du candidat est liée à l'apprentissage, l'âge n'a plus l'effet attendu (tableau 3).

L'analyse des tableaux 2 et 3 nous permet de conclure que ce n'est pas dans toutes les conditions que l'âge est un facteur facilitant l'admissibilité des candi- 
dats. Car, comme nous venons de le voir, la durée de l'expérience, lorsqu'utilisée comme variable contrôle, diminue la pertinence prédictive de la variable âge. De plus, le contrôle de la relation entre l'âge et le degré d'admissibilité par la pertinence de l'expérience nous indique que l'expérience liée à l'apprentissage pourrait être un facteur qui neutralise la variable âge.

En effet, bien plus que l'âge du candidat, c'est ce lien avec l'apprentissage qui augmente les chances du candidat d'être reconnu admissible. La proportion de candidats admissible, lorsque l'expérience est liée à l'apprentissage (tableau 3), est supérieure à celui des candidats dont l'expérience n'est pas liée à l'apprentissage $\left(\mathrm{T}^{2}: \mathrm{A}=53.4 \%\right.$, et $\left.\mathrm{T}^{1}: \mathrm{A}=19.4 \%\right)$. Cette relation statistique est cependant de faible intensité. La proportion de candidats âgés admissibles double si l'expérience est liée à l'apprentissage $\left(\mathrm{T}^{1}: \mathrm{A}=36.1 \%\right.$, et $\left.\mathrm{T}^{2}: \mathrm{A}=63.8 \%\right)$.

Le contrôle de la relation entre l'âge et le degré d'admissibilité avec le niveau scolaire (tableau 4) et les résultats scolaires (tableau 5) nous permet de constater que: le fait d'être âgé de 30 ans et plus augmente les chances d'un candidat d'être reconnu admissible lorsqu'il détient au moins un diplôme d'études collégiales et des résultats scolaires supérieurs à $65 \%$; le fait de détenir au moins un diplôme d'études collégiales (tableau 7) et des résultats scolaires élevés augmente en outre ses chances d'être reconnu admissible.

La relation statistique significative entre l'âge et le degré d'admissibilité n'est pas modifiée lorsque nous introduisons la variable niveau de scolarité (tableau 4). Cependant, l'intensité de la relation est plus du double pour la population ayant au moins un diplôme d'études collégiales $\left(T^{1}: R=0.35\right.$ et $\left.T^{2}: R=0.61\right)$. Dans le cas également de la population la plus âgée et la plus scolarisée, presque la totalité d'entre elle se retrouve dans la catégorie admissible $\left(\mathrm{T}^{2}: \mathrm{A}=85.7 \%\right)$. La proportion de candidats âgés admissibles est quatre fois plus grande si le niveau de scolarité est au moins égal à la détention d'un diplôme d'études collégiales $\left(\mathrm{T}^{\mathrm{I}}: \mathrm{A}\right.$ $=26.7 \%$ et $\mathrm{T}^{2}: \mathrm{A}=85.7 \%$ ).

L'introduction de la variable niveau de scolarité ne modifie donc pas la relation primitive. Elle la spécifie cependant en accentuant ce facteur: niveau de scolarité supérieure (avec D.E.C.) pour la population la plus âgée.

Lorsque nous contrôlons la relation primitive par les résultats scolaires, nous trouvons (tableau 5) que la relation statistique significative entre l'âge et le degré d'admissibilité n'est plus significative lorsque les résultats scolaires sont faibles (moins de $65 \%$ ). La relation primitive demeure pour des résultats scolaires entre $65 \%$ et $75 \%$ et, de plus de $75 \%$. Nous constatons que l'intensité de la relation augmente avec les résultats scolaires $\left(\mathrm{T}^{2}: \mathrm{R}=0.33\right.$ et $\mathrm{T}^{3}: \mathrm{R}=0.43$ ). Ces variations vont dans le même sens de celles observées pour le niveau scolaire. Comme dans le cas précédent, chez les plus âgés (30 ans et plus), un plus grand nombre de personnes se retrouvent admissibles lorsqu'elles cumulent les résultats scolaires les plus élevés $\left(\mathrm{T}^{3}: \mathrm{A}=78.8 \% \mathrm{~N}-\mathrm{A}=22.2 \%\right.$ ); lorsque les résultats sont entre $65 \%$ et $75 \%$, le pourcentage des admissibles est réduit à $35.6 \%$ et des nonadmissibles à $64.4 \%$. 
Tableau 4: DEGRE DADMISSSIBILIIE DES CANDIDATS ADULTES

( $\$$ ) SELON L 'AGE ET MIYEAU DE SCOLARITE

\begin{tabular}{|c|c|c|c|c|}
\hline \multirow{3}{*}{$\begin{array}{l}\text { WIVE AU DE } \\
\text { SCOL ARITE }\end{array}$} & \multirow[t]{2}{*}{ AGE } & \multicolumn{3}{|c|}{ DEGRE DADMISSIBILITE } \\
\hline & & ADMISSIBLE (\%) & NON ADMISSIELE (\%) & $n$ \\
\hline & 30 anst & 26,7 & 73,3 & 86 \\
\hline \multirow[t]{3}{*}{ ( $\left.I^{\prime}\right)$ SAMS D.E.C } & 29 ans- & 8,1 & 91,9 & 111 \\
\hline & \multirow[t]{2}{*}{ Total 9} & 16,2 & 83.3 & 197 \\
\hline & & $x^{2}=11.04$ & \multicolumn{2}{|l|}{$R=0,35$} \\
\hline \multirow{4}{*}{$\left(T^{2}\right)$ AYEC D.E.C. } & & ADMISSIBLE (\%) & NON ADMISSIBLE (\$) & 3 \\
\hline & 29 ans - & 25,6 & 74,4 & 43 \\
\hline & \multirow[t]{2}{*}{ Total 9} & 55,3 & 44.7 & 85 \\
\hline & & $X^{2}=28,69$ & $R=0,61$ & \\
\hline
\end{tabular}

TobleaU 5: DEGRE DADMISSSIBILITE DES CAMDIDATS ADULTES

(\%) SELON L'AGE ET LES RESULTAIS SCOLAIRES

\begin{tabular}{|c|c|c|c|c|}
\hline \multirow{2}{*}{$\begin{array}{l}\text { RESULT ATS } \\
\text { SCOLAIRES }\end{array}$} & \multirow[t]{2}{*}{ AGE } & \multicolumn{3}{|c|}{ DEGRE DADMISSIBILITE } \\
\hline & & $\begin{array}{c}\text { ADMISSIBLE ( } 8) \\
16,7\end{array}$ & $\begin{array}{c}\text { NON ADMISSIBLE (\$) } \\
83,3\end{array}$ & $\begin{array}{l}n \\
6\end{array}$ \\
\hline \multirow{3}{*}{$\left(T^{\prime}\right)$ MOINS DE $65 \%$} & $\$ 29$ ans- & 0,0 & 100,0 & 28 \\
\hline & \multirow[t]{3}{*}{ Tolal 8} & 2,9 & 97,1 & 34 \\
\hline & & $x^{2}=0,74$ & $R=0,38$ & \\
\hline \multirow{4}{*}{$\left(I^{2}\right)_{65 \%}$ ค75\% } & & $\begin{array}{c}\text { ADMISSIBLE ( }(\Phi) \\
35,6\end{array}$ & $\begin{array}{c}\text { NON ADMISSIBLE (\$8) } \\
64,4\end{array}$ & $\begin{array}{l}n \\
87\end{array}$ \\
\hline & 29 ans- & 8,7 & 91,3 & 92 \\
\hline & Total 9 & 21,8 & 78,2 & 179 \\
\hline & \multirow[b]{2}{*}{30 onst } & $X^{2}=17.49$ & $R=0,33$ & \\
\hline \multirow[t]{4}{*}{$\left(T^{3}\right)_{\text {PLUS DE }} 75 \%$} & & $\begin{array}{c}\text { AOMISSIBLE (\%) } \\
78,8\end{array}$ & $\begin{array}{c}\text { NON ADMISSIQLE (ळ) } \\
22,2\end{array}$ & $\begin{array}{l}n \\
36\end{array}$ \\
\hline & 29 ans- & 35,3 & 64,7 & 34 \\
\hline & \multirow[t]{2}{*}{ Total $\$$} & 57,1 & 42,9 & 70 \\
\hline & & $x^{2}=11,21$ & $R=0,43$ & \\
\hline
\end{tabular}

Donc, l'introduction de la variable résultats scolaires ne modifie pas la relation entre l'âge et le degré d'admissibilité sauf pour le groupe ayant les plus faibles résultats scolaires. Elle la spécifie cependant en accentuant le facteur: résultats scolaires élevés (plus de $75 \%$ ) pour la population plus âgée. La proportion des candidats âgés admissible augmente si les résultats scolaires sont forts $\left(\mathrm{T}^{1}: \mathrm{A}=\right.$ $\left.50 \%, \mathrm{~T}^{2}: \mathrm{A}=80.8 \%, \mathrm{~T}^{3}: \mathrm{A}=100 \%\right)$.

Le contrôle de la relation entre l'âge et le degré d'admissibilité par le niveau scolaire et les résultats scolaires nous permet de dégager (tableaux 6 et 7) que le 
niveau de scolarité est plus prédictif du degré d'admissibilité des candidats que les résultats scolaires.

Ainsi, le fait d'être âgé de 30 ans et plus augmente les chances d'être admissible. Cette tendance est associée cependant à certaines conditions: elle s'observe d'autant plus si la personne postulante a au moins un diplôme de niveau collégial et a obtenu des résultats scolaires élevés, soit de plus de $75 \%$. Nous constatons que la norme minimale pour les personnes de 30 ans et plus est davantage le niveau scolaire que les résultats scolaires.

\section{Discussion et conclusion}

L'analyse des différentes informations recueillies nous a permis de connaître certains critères utilisés pour rendre opérationnelle la politique d'admission des adultes à l'Université Laval en 1980. Elle nous a permis aussi d'identifier les facteurs favorisant l'admissibilité d'un candidat adulte.

En effet, nos résultats mettent en évidence certains éléments favorisant l'admissibilité des candidats adultes lors de la "pré-sélection" effectuée au Service d'accueil. Il s'agit bien de "pré-sélection" car seulement $28 \%$ des individus, évalués par ce Service, sont reconnus admissibles aux études universitaires selon le score obtenu lors de l'évaluation des acquis par l'utilisation de la grille d'évaluation S.E.M.

Comme nous l'avions supposé, l'âge est un facteur favorisant l'admissibilité des candidats. Mais la durée de l'expérience constitue elle aussi un avantage pour les adultes lors du processus d'évaluation des chances de réussite.

Si l'âge du candidat a un effet évident sur le degré d'admissibilité, d'autres éléments nous sont apparus importants lors de ce processus d'évaluation. En effet, posséder des expériences liées à l'apprentissage, au moins un diplôme de niveau collégial et des résultats scolaires supérieurs à $65 \%$ augmentent les chances du candidat d'être reconnu admissible.

Si ces résultats semblent énumérer des évidences inscrites dans la pondération de la grille S.E.M., ils démontrent clairement que l'Université Laval accordait en 1980 une plus grande importance à la performance scolaire antérieure des adultes lors de l'évaluation des chances de réussite du candidat adulte. En effet, le profil de référence, pour l'évaluation de l'adulte, s'apparente à celui de la clientèle traditionnelle où la formation scolaire est principalement considérée. Ceci constitue la principale critique concernant l'assouplissement proposé aux normes d'admission de l'Université Laval pour les adultes. En effet, si le choix des attestations, pour évaluer les chances de succès d'un candidat adulte, est élargi pour inclure les connaissances découlant d'une expérience pertinente, les critères pour juger de ces expériences semblent permettre difficilement de faire valoir des acquis non-formels et non-scolaires.

L'introduction, dans une politique d'admission, du principe de reconnaissance des expériences acquises par un individu constitue un pas vers une plus grande démocratisation des études universitaires. Mais elle pose dans son entier, au niveau de l'application de la politique officielle, le problème de l'évaluation et 

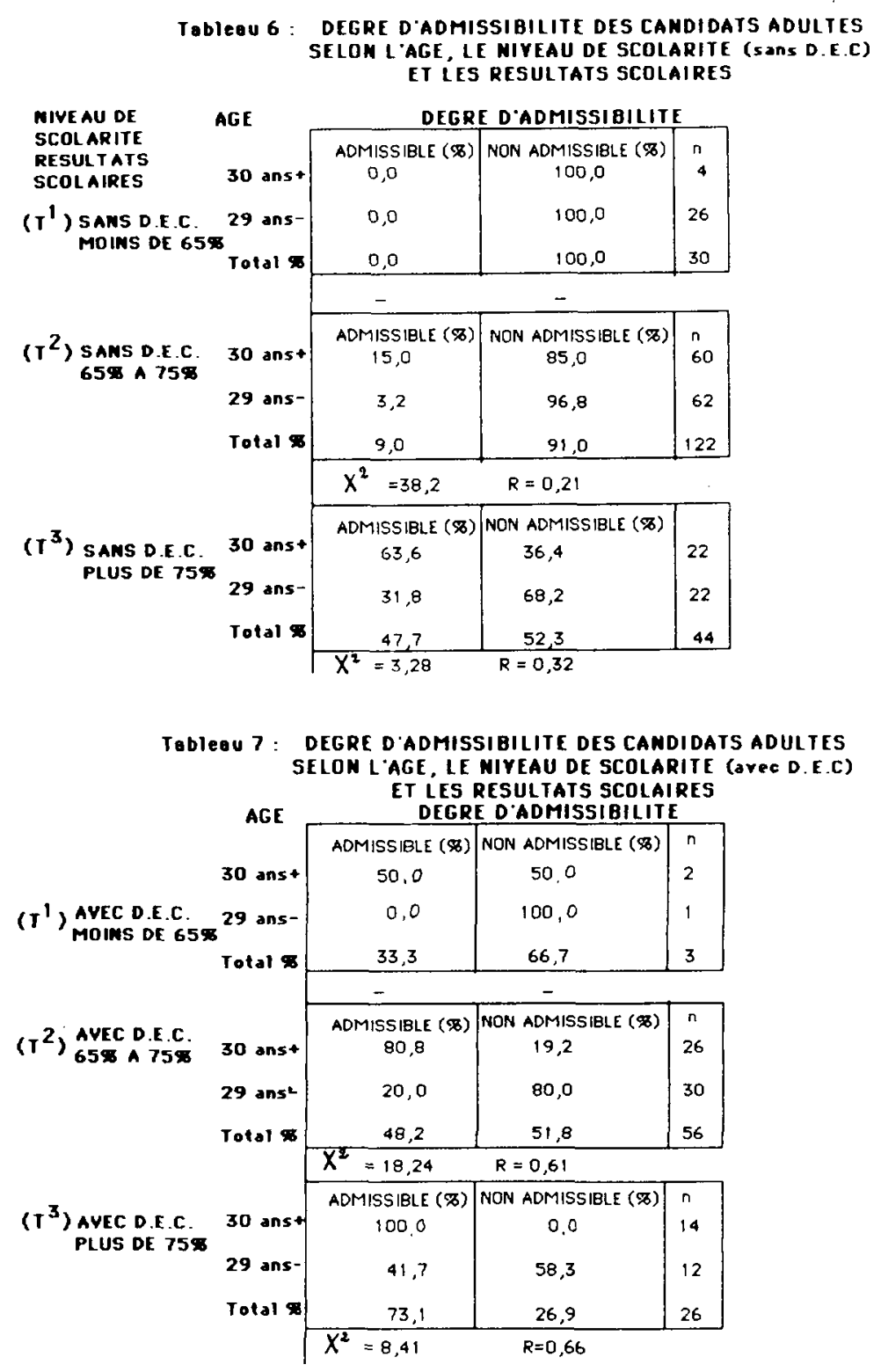

de la mesure des chances de succès des candidats désirant faire reconnaître les connaissances acquises par les expériences diverses.

Dans le cas qui nous intérese, les conseillers du Service d'accueil doivent évaluer ces expériences et juger de leur pertinence. Pour rendre opérationnelle la politique officielle d'admission, ils ont dû établir des critères et ils ont construit 
un outil (grille S.E.M.) afin de disposer d'un canevas de base pour l'évaluation des acquis des adultes.

Selon le rapport Lauzon (1981), une expérience pertinente est une expérience liée à l'apprentissage. Notre étude institutionnelle met clairement en évidence le fait suivant: pour être jugée pertinente aux yeux de l'Université Laval, l'expérience doit par "nature avoir pu contribuer à la formation, à la culture, à la maturité qui sont des facteurs de cette possibilité globale de réussir au niveau universitaire" (Lauzon, 1981: 48). L'Université Laval mesure ainsi la pertinence de l'expérience et le type d'apprentissage. Selon le directeur du Service d'accueil, l'expérience pertinente est celle liée au champ d'études envisagé et permettant à la personne d'atteindre un certain développement intellectuel. L'analyse documentaire des textes concernant l'application de la politique d'admission des adultes précise que le développement intellectuel d'un candidat adulte se traduit par "une connaissance de base de l'enseignement supérieur, qu'il a l'habitude des travaux, mémoires, rapports et dissertations, qu'il possède par ailleurs une méthode de travail s'appliquant à des études de type intellectuel, et enfin qu'il possède une démarche scientifique rigoureuse" (Pelletier, 1979: 6). Pour l'Université Laval, des acquis expérientiels tels: session de perfectionnement, crédits de l'Extension de l'enseignement et expérience de travail dans un domaine connexe au choix envisagé seront probablement considérés favorables lors 'de l'évaluation des dossiers de l'adulte (Université Laval 1981: 79).

Lors de ce processus d'évaluation du dossier des adultes, les agents d'admission jugent de la pertinence de l'expérience des adultes en cherchant jusqu'à quel point ces expériences ont permis à la personne d'atteindre un certain développement intellectuel, nécessaire au candidat pour entreprendre et réussir des études universitaires. S'ils établissent que le lien entre l'apprentissage acquis par le biais de l'expérience et celui nécessaire aux études universitaires est étroit, le candidat verra augmenter ses chances d'être reconnu admissible aux études universitaires.

Toutefois, nos analyses nous ont permis de constater l'imprécision de l'outil d'évaluation utilisé en regard des expériences non-scolaires et non-formelles. Cette imprécision a pour effet principal de limiter l'accès aux études universitaires aux adultes dont la formation s'est moins réalisée dans le réseau scolaire.

Comme nous l'avons observé, d'autres facteurs sont importants lors du processus d'évaluation en vue de déterminer le degré d'admissibilité du candidat adulte. Ces facteurs sont les acquis académiques (diplôme et résultats). Une bonne performance scolaire antérieure des adultes augmente les chances des candidats d'être reconnus admissibles aux études universitaires. En regard de l'accessibilité à l'enseignement supérieur, le maintien de normes, presque exclusivement de nature académique, pénalise plusieurs adultes en exigeant de ces derniers une trop longue période de rattrapage scolaire. Car, aux yeux de l'institution universitaire, une bonne performance scolaire antérieure semble presque la seule garantie de la réussite d'un candidat dans les études qu'il désire entreprendre.

En considérant nos résultats, le profil de référence, pour la "pré-sélection" des adultes, est principalement la formation scolaire et sa dimension cognitive. Les exigences habituellement reconnues pour l'admission de la clientèle régulière 
(D.E.C., profil d'accueil) demeurent donc les principaux critères pour juger des chances de succès de la clientèle adulte et l'admettre sans condition.

Le concept d'expérience de vie semble être utilisé de façon secondaire, comme confirmation au renforcement des succès scolaires antérieurs, plutôt que comme critère autonome. Rappelons ici que nous n'avons observé que les adultes possédant des acquis scolaires similaires aux normes officielles d'admission pour la clientèle régulière et des expériences pertinentes sont pour la plupart reconnus admissibles aux études universitaires. Cela nous a peu étonnés. Ce qui nous a surpris cependant, c'est la marge étroite des.admissibles parmi ceux cumulant des acquis expérientiels non-scolaires et non-formels. La politique officielle d'admission des candidats adultes laissait supposer une plus grande accessibilité à l'Université aux candidats n'ayant pas complété ces acquis académiques. Malgré les termes de cette politique d'admission, adhérant au principe de la reconnaissance des acquis expérientiels, la majorité des adultes présentant un profil de type expérientiel est reconnue non admissible aux études universitaires (près de $72 \%$ ).

Une ouverture de l'Université à la clientèle adulte ne sera importante que lorsqu'on aura trouvé une façon de relier l'expérience de vie au succès scolaire universitaire lui-même et non aux succès scolaires antérieurs. Pour cela, il faudra davantage faire des expérimentations et en mesurer systématiquement les efforts.

Il faudrait aussi solliciter les facultés pour qu'elles précisent le contenu des cours offerts dans leurs programmes afin de fournir aux conseillers, qui évaluent le dossier des adultes, un guide détaillé des connaissances et des savoirs dispensés. L'adéquation entre les acquis non-formels et non-scolaires et ce qui est enseigné serait ainsi facilitée et permettrait une évalution plus éclairée des acquis du candidat adulte.

\section{RÉFÉRENCES}

Conseil de l'Université Laval, 1973, Résolution U-73-334, Université Laval, Québec, 11 décembre 1973.

Conseil de l'Université Laval, 1974, Résolution U-74-130, Université Laval, Québec, 25 février 1974.

Darling, A.L., "The admission of non-matriculated adults to university credit courses" in The Canadian Journal of Higher Education, XV (2) 1985, 91-97.

Héon, L., La reconnaissance des acquis expérientiels dans la politique d'admission des adultes à l'Université Laval. Thèse de maîtrise, Université Laval, septembre 1984.

Lauzon, M., L'accueil de l'adulte à l'Université Laval. Bureau de la recherche institutionnelle, Université Laval, Québec, février 1981.

Leblanc, N., L'Université Laval au-devant de l'éducation permanente. Université Laval, Québec, décembre 1973.

Pelletier, G.A., "Le Service d accueil", exposé prononcé dans le cadre des réunions d'information de la Forêt Montmorency, Université Laval, Québec, octobre 1979.

Rapport Parent, Rapport de la Commission royale d'enquête sur l' enseignement dans la Province de Québec. Tome 1, Québec, Gouvernement du Québec, 1963-1966.

Service d'accueil, "Grille S.E.M. pour l'évaluation des acquis", document pour usage interne, Université Laval, Québec, février 1979.

Université Laval, Projet Laval: L'accès permanent à l'Université Laval, Université Laval, Québec avril 1981 . 